BULLETIN OF THE

AMERICAN MATHEMATICAL SOCIETY

Volume 81, Number 1, January 1975

\title{
NEW INFINITE FAMILIES IN THE STABLE HOMOTOPY OF SPHERES
}

\author{
BY LARRY SMITH ${ }^{1}$
}

Communicated by Edgar Brown, Jr., July 2, 1974

Let $p$ be an odd prime and denote by $\pi^{s}$ the stable homotopy ring of spheres. There are known two infinite families of elements

$$
\alpha_{t} \in \pi_{2 t(p-1)-1}^{s}, \quad \beta_{t} \in \pi_{2 t\left(p^{2}-1\right)-2 p}^{s}
$$

introduced in [4] and [2I]; the latter sequence only when $p>3$. In [5] Toda also constructed elements

$$
\epsilon_{i} \in \pi_{2(p-1)\left(p^{2}+i\right)-2}^{s} \quad i=1, \cdots, p-1,
$$

and by a seemingly numerical accident in Toda's tables (at least up to a nonzero multiple) $\epsilon_{p-1}=\beta_{p}$. Thus the element $\epsilon_{p-1}$ has a bordism theoretic interpretation. In this note we announce the results of our investigation of the remaining elements $\epsilon_{1}, \cdots, \epsilon_{p-2}$. To describe our results we recall some notations and results of [2].

Let $\Omega_{*}^{U}()$ denote the complex bordism homology theory [3]. Recall that the coefficient ring is

$$
\Omega_{*}^{U} \simeq \mathrm{Z}\left[x_{2}, x_{4}, \cdots\right] ; \quad \operatorname{deg} x_{2 i}=2 i
$$

an infinite polynomial ring. Polynomial generators of dimension $2 p^{i}-2$ play a special role in the theory, are called Milnor manifolds, and are usually denoted by $\mathbf{V}^{2 p^{i-2}}$.

Following the stable notations of [2] let $V(0)=S^{0} \cup_{p} e^{1}$, so that $\widetilde{\Omega}_{*}^{U}(V(0)) \simeq \widetilde{\Omega}_{*}^{U} /(p)$. There is the map [2I; 1.5] $\bar{\varphi}: S^{2 p-2} V(0) \rightarrow V(0)$ such that $\bar{\varphi}_{*}: \widetilde{\Omega}_{*}^{U}\left(S^{2 p-2} V(0)\right) \rightarrow \widetilde{\Omega}_{*}^{U}(V(0))$ is given by

$$
\bar{\varphi}_{*}\left(\Sigma^{2 p-2} \gamma_{0}\right)=[\mathrm{CP}(p-1)] \gamma_{0},
$$

where $\gamma_{0} \in \widetilde{\Omega}_{0}^{U}(V(0))$ is a generator. There is the iteration $\overline{\varphi^{s}}: S^{2 s(p-1)} V(0)$ $\rightarrow V(0)$ whose mapping cone we denote by $V^{(s)}(1)$. (N.B. when $s=1$

AMS (MOS) subject classifications (1970). Primary 55E40, 57D90.

${ }^{1}$ Research supported by NSF GP 38967. 
$V^{(1)}(1)$ is just the space $V(1)$ of [2I].

Our first result describes the elements $\epsilon_{1}, \cdots, \epsilon_{p-1}$ in bordism theoretic terms.

THEOREM 1. Let $p>3$ be a prime. Then there exists a stable map

$$
\hat{\epsilon}_{r}: S^{2 p\left(p^{2}-1\right)} \rightarrow V^{(p-r)}(1)
$$

such that

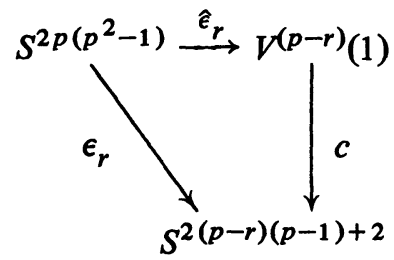

is commutative. (N.B. The cell structure of $V^{(p-r)}(1)$ is $S^{0} \cup_{p} e^{1} \cup$ $e^{2(p-r)(p-1)+1} \cup_{p} e^{2(p-r)(p-1)+2}$, and $c$ is collapse onto the top cell.)

Moreover

$$
\left[S^{2 p\left(p^{2}-1\right)}, \hat{\epsilon}_{r}\right]=\left(\left[V^{2 p^{2}-2}\right]^{p}+[M]\right) \gamma_{0} \in \widetilde{\Omega}_{*}^{U}\left(V^{(p-r)}(1)\right),
$$

where $[M] \in\left([\mathrm{CP}(p-1)]^{p-r-1}\right) \subset \Omega^{U} * \square$

Note that for $r=p-1$, recalling $\epsilon_{p-1}=\beta_{p}$, we see that Theorem 1 is contained in [2I, §5]. This serves as the starting point for the proof which is completed by very careful use of the calculations of Toda.

The next result advances us to the second stage of the program of [2I] for producing infinite families in $\pi_{*}^{s}$.

THEOREM 2. Let $p>3$ be a prime. Then there is an extension of $\hat{\epsilon}_{r}$ to $V^{(p-r)}(1)$, that is, there exists a stable map such that the diagram

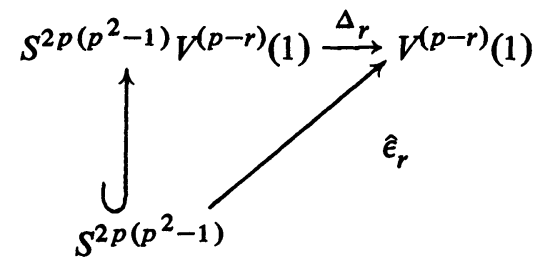

is commutative.

Recalling the main result of [2], we now define

$$
\epsilon_{r}(t) \in \pi^{s}{ }_{2(p-1)}\left[t p^{2}+(t-1) p+r\right]-2,
$$


where $r=1, \cdots, p-1$, and $t>0$, by the commutativity of the diagram

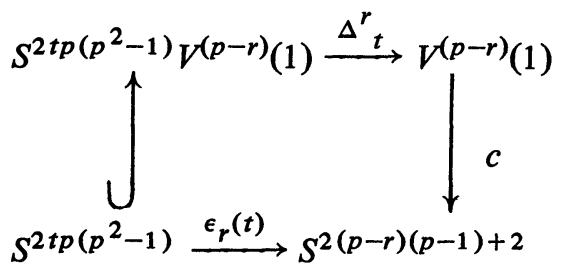

and obtain

THEOREM 3. Let $p>3$ be a prime. Then the elements

$$
\epsilon_{r}(t) \in \pi_{2(p-1)\left[t p^{2}+(t-1) p+r\right]-2}^{s}\left\{\begin{array}{l}
r=1, \cdots, p-1, \\
t>0
\end{array}\right.
$$

are all nonzero of order $p$. Moreover $\epsilon_{r}(1)=\epsilon_{r}$ and $\epsilon_{r}(p-1)=\beta_{r p}$. Thus each element $\epsilon_{1}, \cdots, \epsilon_{p-1}$ starts an infinite family in $\pi_{*}^{s}$.

Note that the mapping cones of $\Delta^{r}{ }_{t}$ provide new examples of cyclic modules over $\Omega^{U}$. that are realizable.

Complete details will appear as part IV of the series [2].

\section{REFERENCES}

1. P. E. Conner and L. Smith, On the complex bordism of complexes with few cells, J. Math. Kyoto Univ. 11 (1971), 315-356. MR 45 \#7722.

2. L. Smith, On realizing complex bordism modules. I, II, III, Amer. J. Math. 92 (1970), 793-856; ibid 93 (1971), 226-263; ibid. 94 (1972), 875-890. MR 43 \#1186a,b; 46 \#10014.

3. R. E. Stong, Notes on cobordism theory, Princeton Univ. Press, Princeton, N. J., Univ. of Tokyo Press, Tokyo, 1968. MR 40 \#2108.

4. H. Toda, p-primary components of homotopy groups. IV. Compositions and toric constructions, Mem. Coll. Sci. Univ. Kyoto Ser. A. Math. 32 (1959), 297-332. MR 22 \#1906.

5. - An important relation in homotopy groups of spheres, Proc. Japan Acad. 43 (1967), 839-842. MR 37 \#5872.

DEPARTMENT OF MATHEMATICS, UNIVERSITY OF VIRGINIA, CHARLOTTESVILLE, VIRGINIA 22901 